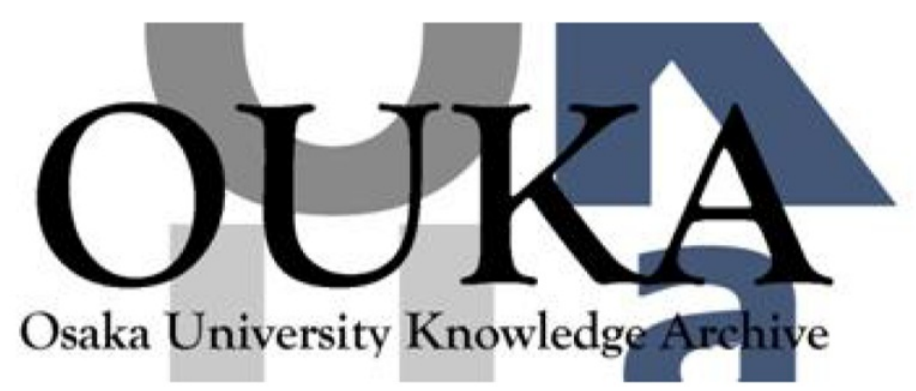

\begin{tabular}{|c|c|}
\hline Title & $\begin{array}{l}\text { Elastic constants of langasite and alpha quartz } \\
\text { at high temperatures measured by antenna } \\
\text { transmission acoustic resonance }\end{array}$ \\
\hline Author (s) & Nakamura, N. ; Sakamoto, M.; Ogi, H. et al. \\
\hline Citation & $\begin{array}{l}\text { Review of Scientific Instruments. 83(7) } \\
\text { p. } 073901-1-\text { p. } 073901-7\end{array}$ \\
\hline Issue Date & $2012-07-05$ \\
\hline oaire:version & VoR \\
\hline URL & https://hdl. handle. net/11094/84237 \\
\hline rights & $\begin{array}{l}\text { This article may be downloaded for personal use } \\
\text { only. Any other use requires prior permission } \\
\text { of the author and AIP Publishing. This article } \\
\text { appeared in Review of Scientific Instruments, } \\
83(7) \text {, } 073901 \text { (2012) and may be found at } \\
\text { https://doi.org/10.1063/1.4731657. }\end{array}$ \\
\hline Note & \\
\hline
\end{tabular}

Osaka University Knowledge Archive : OUKA

https://ir. Library. osaka-u. ac. jp/

Osaka University 


\title{
Elastic constants of langasite and alpha quartz at high temperatures measured by antenna transmission acoustic resonance
}

\author{
N. Nakamura, M. Sakamoto, H. Ogi, ${ }^{\text {a) }}$ and M. Hirao \\ Graduate School of Engineering Science, Osaka University, Toyonaka, Osaka 560-8531, Japan
}

(Received 25 April 2012; accepted 13 June 2012; published online 5 July 2012)

\begin{abstract}
A method for measuring elastic constants of piezoelectric materials at high temperature up to $1224 \mathrm{~K}$ is proposed. It determines all independent elastic constants by measuring resonance frequencies of a rectangular parallelepiped piezoelectric specimen contactlessly using its own piezoelectricity with an antenna. Without using conventional contacting piezoelectric transducers, vibrational sources are excited directly in the specimen by the oscillating electric field. Capability of the method is demonstrated by measuring the elastic constants of langasite at high temperature up to $1224 \mathrm{~K}$, and temperature coefficients of the elastic constants are determined. In addition, elastic constants of alpha quartz are measured at high temperature up to just below the alpha-beta phase transition temperature. Considering the local deformation with temperature increment, an interpretation based on the strain energy reduction is proposed for the unusual temperature dependence of $C_{66}$. Furthermore, the internal-friction tensor is measured, and the relationship between the observed anisotropy in internal friction and the structural evolution with temperature increment is discussed. (9 2012 American Institute of Physics. [http://dx.doi.org/10.1063/1.4731657]
\end{abstract}

\section{INTRODUCTION}

Elastic constants of piezoelectric material are essential parameters for designing acoustic sensors and filters. Because the temperature at which sensors are used is wideranging, temperature dependence of elastic constants have been required to develop optimized sensors at the operating temperature. However, the measurement has never been straightforward. One of the reasons is the low crystallographic symmetry of piezoelectric materials; for example, langasite $\left(\mathrm{La}_{3} \mathrm{Ga}_{5} \mathrm{SiO}_{14}\right)$ and $\alpha$-quartz show trigonal symmetry (six independent elastic constants) and typical metals such as copper and iron show cubic symmetry (three independent elastic constants). As symmetry becomes lower, the number of independent elastic constant increases. It makes measurement of elastic constants difficult, especially at high temperatures. There are several methods that measure elastic constants of piezoelectric materials: plate-thickness resonance, ${ }^{1,2}$ the rod vibration, ${ }^{3}$ and pulse-echo. ${ }^{4}$ These methods require several specimens with different crystallographic orientations to determine a complete set of elastic constants, and each specimen has to be examined individually. Then, orientation error and dimension error of each specimen are accumulated in the resultant elastic constants, and as the number of specimens increases, the resultant error increases. Also, it has to be noted that for measuring the temperature dependence of elastic constants, experiments at several temperatures have to be carried out for each specimen, which is highly time-consuming. These features indicate that the conventional methods are not optimized for studying temperature dependence of elastic constants.

Among the conventional methods, resonant ultrasound spectroscopy (RUS) is a candidate for the high-temperature

a)Electronic mail: ogi@me.es.osaka-u.ac.jp. experiment, because it can determine all independent elastic constants from a single specimen by a single frequency sweep. ${ }^{5,6}$ This is an advantage over other methods, and the RUS method has been already applied to temperature dependence of elastic constants of various materials. ${ }^{7-9}$ However, in the conventional RUS method, there is a difficulty to be solved for high-temperature measurements. RUS method measures resonance frequency of mechanical free vibrations of a specimen, and determines a set of elastic constants inversely from a number of resonance frequencies. Resonance frequencies are generally measured by holding a specimen between two piezoelectric transducers. Because piezoelectricity of the transducers disappears at elevated temperature, conventional transducers cannot be used for high-temperature measurements. Although the methods to use buffer rods connecting the piezoelectric transducers and specimen were proposed for high-temperature measurements, ${ }^{7,8}$ separation of the rod's signal from the specimen's signal is problematic. It is also inevitable that holding a specimen by the transducers obstructs free vibrations and consequently degrades measurement accuracy of resonance frequencies. ${ }^{10}$

Antenna transmission acoustic resonance (ATAR) is here proposed for solving the difficulty and for achieving hightemperature measurement of elastic constant of piezoelectric materials. Measurement principle of the ATAR method is the same as that of RUS method, but the experimental setup is different; resonance frequencies are now measured owing to the specimen's own piezoelectricity using an antenna, instead of the piezoelectric transducers. The antenna applies no external force except for the specimen's mass, and it achieves ideal free vibrations of the specimen.

In this study, we show the capability of the ATAR method by measuring the elastic constants of langasite and $\alpha$-quartz. Langasite shows higher electromechanical coupling coefficients than $\alpha$-quartz. Because piezoelectricity exists 
until the melting point $(1743 \mathrm{~K})$, langasite is a candidate for high-temperature transducers and sensors. Temperature dependence of elastic constants has been reported up to $620 \mathrm{~K}$ by the RUS method ${ }^{11}$ and up to $1173 \mathrm{~K}$ by the rod vibration method. ${ }^{3}$ Here we measure the elastic constant up to $1224 \mathrm{~K}$, and demonstrate capability of the ATAR method.

Also, $\alpha$-quartz is measured. Elastic constants are measured up to just below the $\alpha-\beta$ phase transition temperature (846 K); we discuss significant differences from the previously reported values. Among the elastic constants, only $C_{66}$ shows anomalous behavior. An interpretation, which is established based on the local strain caused by thermal expansion, is proposed for the anomaly. Also, temperature dependence of the internal friction tensor is determined, which has never been reported at high temperatures. In the internal-friction measurement, an experimental setup that minimizes energy loss caused by other factors is required. The antenna causes the limited contact with the specimen, and it allows us to measure the internal friction. Our measurement reveals that an internal friction component $Q_{33}$ shows anomalous behavior just below the transition temperature.

\section{MEASUREMENTS}

\section{A. Specimen}

Langasite and $\alpha$-quartz belong to the point group 32. They show trigonal symmetry, and possess six independent elastic constants, two piezoelectric constants, and two dielectric constants. A rectangular parallelepiped specimen was prepared for each single crystal. Specimen sizes were 8.049 $\times 4.061 \times 3.958 \mathrm{~mm}^{3}$ for langasite and $4.768 \times 5.234$ $\times 5.797 \mathrm{~mm}^{3}$ for $\alpha$-quartz. The dimensions were measured using a micrometer. Mass density was calculated from the dimensions and mass measured using an electric balance, and their values were 5677 and $2645 \mathrm{~kg} / \mathrm{m}^{3}$, respectively.

\section{B. Antenna transmission acoustic resonance}

Figure 1 shows a diagram of the antenna developed in the present study. The antenna consists of three chromel wires. An end of each wire is curled so as to roughly surround the specimen. The wires are placed side by side with a small gap between them, and the curled regions are covered with cement paste. It forms a cement tube that the curled wires are embedded in the circumferential direction inside the tube's

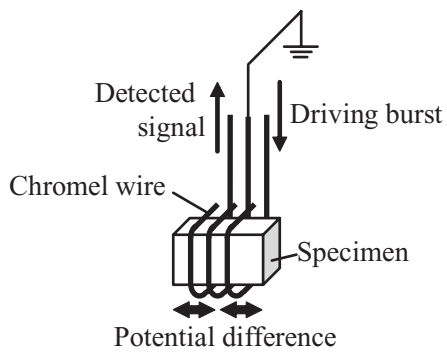

FIG. 1. Schematic image of the antenna for measuring resonance frequency of a piezoelectric specimen. The cement tube that is explained in the text is not shown here.

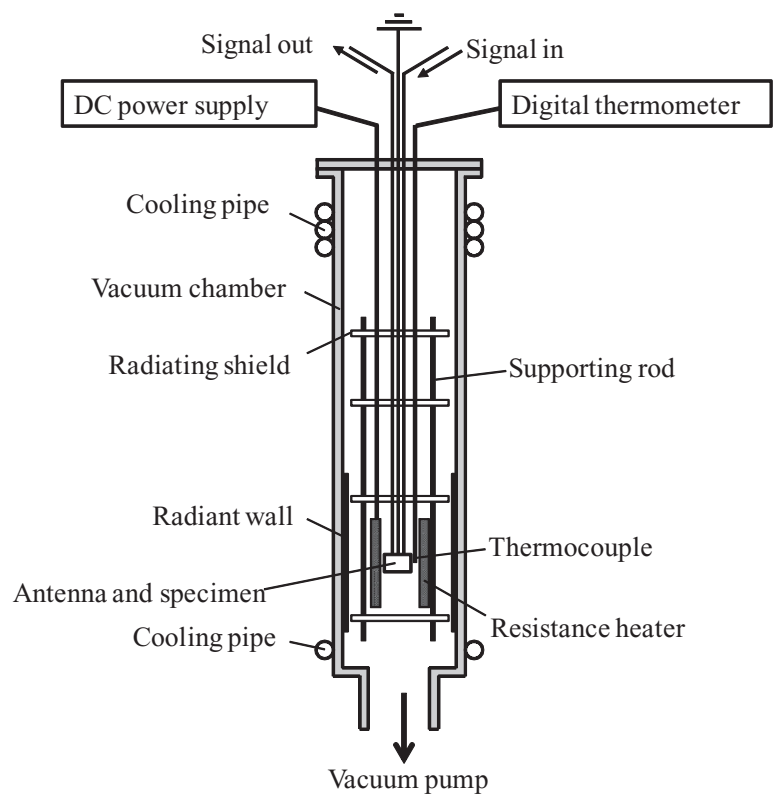

FIG. 2. Cross-sectional view of the vacuum chamber.

wall. The cement is then solidified. Resonance frequencies are measured by placing a specimen inside the ceramic tube. Inner diameter of the tube is slightly larger than the specimen's dimensions, and the specimen does not touch the inner surface of the tube except at the specimen's bottom. This realizes nearly ideal resonance vibrations. A thermocouple is located beside the specimen. The tube and the thermocouple are surrounded by a resistive heater. All of them are placed in a vacuum chamber, and resonance spectra are measured. Configuration of the vacuum chamber is shown in Fig. 2. The pressure during the experiments was between $4.5 \times 10^{-3}$ and $1.6 \times 10^{-2} \mathrm{~Pa}$ for langasite and between $5.3 \times 10^{-3}$ and 9.3 $\times 10^{-2}$ Pa for $\alpha$-quartz.

One of the chromel wires is connected with the ground. One of the remaining wires is connected to a synthesizer through a gated amplifier for excitation purpose, and the other is connected to a superheterodyne spectrometer through a preamplifier for detection purpose. To excite vibration in a specimen, a sinusoidal tone burst is applied to the transmission wire. Potential difference between the transmission wire and the ground wire excites the electric field in the normal direction to the straight line of the wires. By inverse piezoelectricity, the field causes oscillation of the specimen at the same frequency of the burst. The oscillating specimen then inversely excites an electric field near the surface through the piezoelectricity, which is picked up by the detecting wire. Amplitude of the detected signal is proportional to the oscillation amplitude of the specimen, and by sweeping the frequency of the driving burst, a resonance spectrum is obtained. When the frequency of the driving burst corresponds to a resonance frequency of the specimen, a high-amplitude vibration occurs and a resonance peak appears in the spectrum. Resonance frequencies are determined by the Lorentz-function fitting method. The antenna transmitting system possesses advantages that electrodes are not required to be deposited on specimen surfaces and no external forces except for its weight are applied. These 
advantages allow us to measure resonance frequencies accurately. The efficiency can be seen in the literature, where the antenna transmitting system was applied to the immunoassay sensor. $^{12,13}$

In the ATAR method, elastic constants are determined in the same manner as the RUS method. ${ }^{14}$ Resonance frequencies of a piezoelectric rectangular parallelepiped specimen depend on the dimensions, mass density, elastic constants, piezoelectric constants, dielectric constants, and crystal orientation. Therefore, elastic constants are determined by measuring the resonance frequencies and the other parameters. Regarding the dimensions and the mass density, they are measured at room temperature first; variations with temperature increment are estimated from the reported temperature dependence of lattice parameters. ${ }^{15,16}$

Regarding the piezoelectric coefficients and dielectric constants, the values given at room temperature are used over the whole temperature range. In high-frequency (ultrasonicfrequency) measurements, the material is stiffened because of suppression of total strain by the piezoelectric strain. This is called piezoelectric stiffening, and the apparent elastic constant $C^{\prime}$ is roughly expressed as $C^{\prime}=C+e^{2} / \epsilon$, where $C$ is the elastic constant, $e$ the piezoelectric constant, and $\epsilon$ the dielectric constant. The piezoelectric stiffening term $e^{2} / \epsilon$ is usually much smaller than the elastic constant $C$. ATAR and RUS methods can principally determine all independent elastic constants and piezoelectric constants from the resonance frequencies. However, measurement of the resonance frequencies at high temperature is difficult, and the number is not enough to determine the piezoelectric constants accurately. For example, in the present study, the number of measurable resonance frequencies is about 20 close to the transition temperature of $\alpha$-quartz. Therefore, the piezoelectric stiffening term was often ignored, $e^{2} / \epsilon=0$, in the determination of elastic constants. ${ }^{7}$ However, the piezoelectric stiffening effect has to be considered in accurate evaluation of the elastic constants. For evaluating the contribution, before hightemperature experiments, we first determined two sets of room-temperature elastic constants by the ATAR method; one was determined with considering the piezoelectric stiffening and the other was determined without it. In the calculation, the reported piezoelectric constants and dielectric constants at room temperature were used (the values are shown in the next paragraph). Then, the difference between the elastic constants of langasite was less than $1.1 \%$ for the diagonal components and was less than $3.3 \%$ for the off-diagonal components. The same analysis was carried out for $\alpha$-quartz. Then, the difference was $8.5 \%$ for $C_{12}$ and was less than $1.5 \%$ for other components. Because these differences are not necessarily small, the piezoelectric stiffening is considered in the analysis using the reported values of $e_{i j}$ and $\epsilon_{i j}$ at room temperature. Effect of temperature dependence of $e_{i j}$ and $\epsilon_{i j}$ on the resultant elastic constant was also evaluated using their temperature dependence reported in previous studies., ${ }^{3,17-19}$ Then, the possible error is $3.9 \%$ for $C_{13}$ and less than $0.8 \%$ for other components in $\alpha$-quartz, and less than $1.2 \%$ for all components in langasite. These contributions are ignored in the present study.

For langasite, piezoelectric constants and dielectric constants reported by Ogi et al., ${ }^{14} e_{11}=-0.397 \mathrm{C} / \mathrm{m}^{2}, e_{14}$
$=0.203 \mathrm{C} / \mathrm{m}^{2}, \epsilon_{11} / \epsilon_{0}=19.04$, and $\epsilon_{33} / \epsilon_{0}=50.51$, are used. Here, $\epsilon_{0}$ denotes the vacuum permittivity. For $\alpha$-quartz, piezoelectric constants reported by Ogi et al., ${ }^{20} e_{11}=0.151$ $\mathrm{C} / \mathrm{m}^{2}$ and $e_{14}=-0.061 \mathrm{C} / \mathrm{m}^{2}$, and averaged value of dielectric coefficients given by Mason ${ }^{21}$ and Koga etal., ${ }^{1} \epsilon_{11} / \epsilon_{0}$ $=4.50$ and $\epsilon_{33} / \epsilon_{0}=4.60$, are used.

\section{RESULTS AND DISCUSSION}

\section{A. Langasite}

Figure 3 shows resonance spectra measured at several temperatures. Each resonance spectrum was measured keeping the target temperature with the fluctuation less than $\pm 1.5^{\circ} \mathrm{C}$. At room temperature, 48 resonance frequencies were identified between 0.1 and $0.9 \mathrm{MHz}$. As the temperature increases, some resonance peaks disappeared, and the minimum number was 25 around $973 \mathrm{~K}$. At $1224 \mathrm{~K}, 35$ frequencies were measurable, which is a large enough number to determine six independent elastic constants. This result confirms capability of the antenna at high temperature, and it is expected that the antenna operates above $1273 \mathrm{~K}\left(=1000^{\circ} \mathrm{C}\right)$.

Figure 4 shows the temperature dependences of elastic constants obtained in the present study. Figure 5 shows Young's moduli $E_{a}$ and $E_{c}$, bulk modulus $B$, and Poisson's ratios $v_{12}, v_{13}$, and $v_{31}$ calculated from the measured elastic constants $\left(v_{i j}=-s_{i j} / s_{i i}\right.$, where $s_{i j}$ denotes the elastic compliance). Elastic constants decrease almost linearly as the temperature increases. This behavior is similar to the observation between 280 and $625 \mathrm{~K}$ measured by Schreuer et al. ${ }^{11}$ with a RUS method. A linear function $C_{i j}(T)=\left[1+a_{i j}(T-293)\right] C_{i j}^{293 \mathrm{~K}}$ was fitted to each temperature dependence between 293 and $1224 \mathrm{~K}$, and the first-order temperature coefficients $a_{i j}$ were deduced. They are listed in Table I together with $C_{i j}$ at 293 and $1224 \mathrm{~K}$. $C_{14}$ shows the largest dependence $\left(a_{14}=-2.66 \times 10^{-4} \mathrm{~K}^{-1}\right)$, and the others show comparable values $\left(a_{i j} \approx-1.0 \times 10^{-4} \mathrm{~K}^{-1}\right)$. At high temperatures, solids generally show a linear negative slope

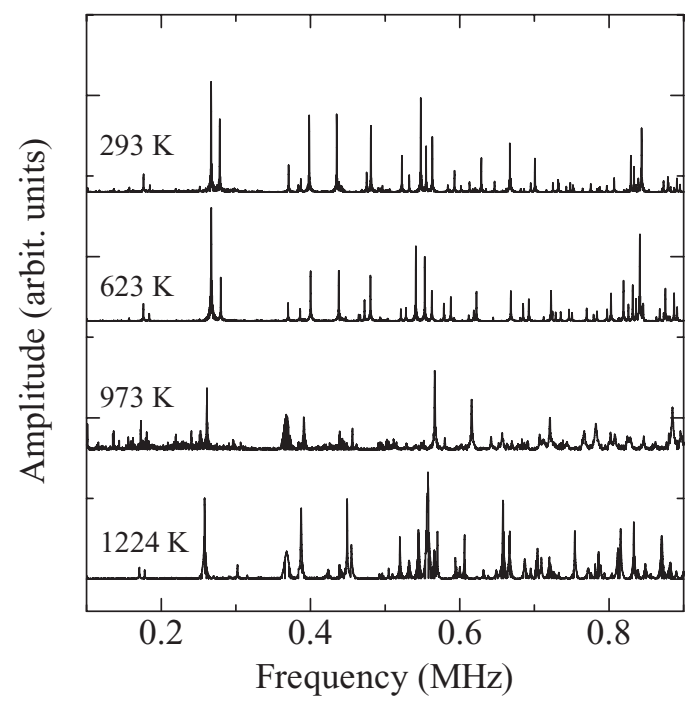

FIG. 3. Resonance spectra of a langasite specimen measured with the antenna method at several temperatures. 


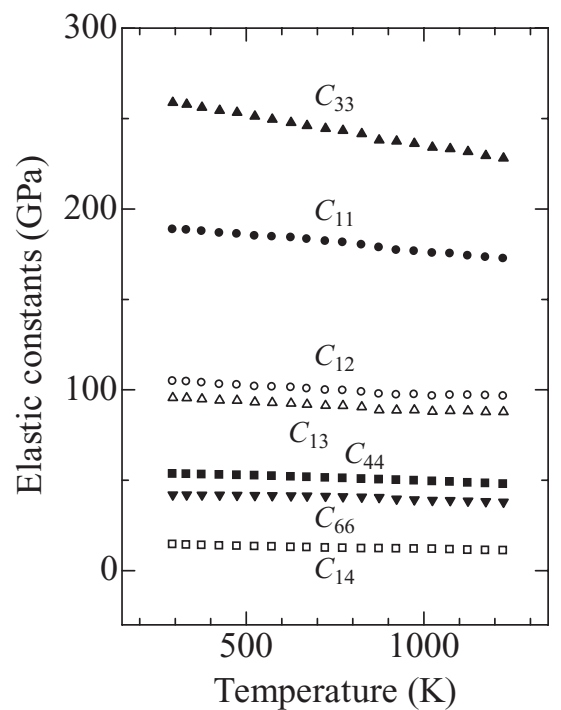

FIG. 4. Temperature dependence of elastic constants of langasite.

in temperature dependence of $C_{i j}$ because of phonon effects. Langasite shows high melting point and no phase transition until the melting. Therefore, we expect that the phonon effect is the primary reason of the linear temperature dependence in the examined temperature range. Regarding Poisson's ratios, they are almost independent of temperature. Considering comparable $a_{i j}$, the independences are reasonable, because Poisson's ratio is expressed as a fraction of homogeneous polynomials of $C_{i j}$ and changes in $C_{i j}$ is canceled in Poisson's ratio. Although $a_{14}$ is not comparable to others, value of $C_{14}$ is small and contribution on Poisson's ratio is expected to be smaller than that of others. We consider that the comparable $a_{i j}$ is a feature that characterizes elastic property of langasite.

Comparing with the reported elastic constants, the present results are close to those given by Schreuer et al., ${ }^{11}$ but are different from the results measured with the rod vibrating method. ${ }^{3}$ The difference should be attributed to the inaccuracy in the previous experiments described in the Introduction.

\section{B. Quartz}

Figure 6 shows the temperature dependence of the elastic constants. Figure 7 shows the temperature dependence of Young's moduli, bulk modulus, and Poisson's ratios calculated from the measured $C_{i j}$. In Fig. 6, present values show

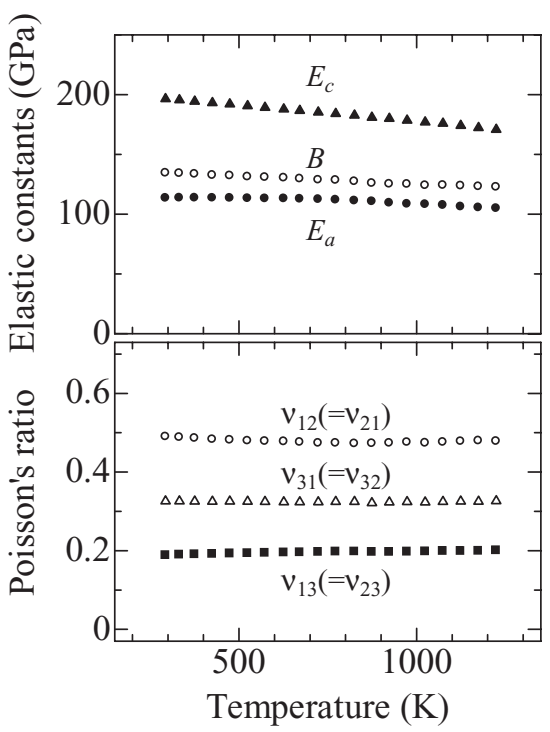

FIG. 5. Temperature dependence of Young's moduli $E$, bulk modulus $B$, and Poisson's ratios $v$ of langasite.

the similar temperature dependence with the values reported by Ohno et al. ${ }^{7}$ as temperature increases, $C_{11}, C_{12}, C_{13}, C_{33}$, and $C_{44}$ decrease, $C_{66}$ increases, and $C_{14}$ slightly decreases just above ambient temperature and it then increases. However, the values disagree, especially near the $\alpha-\beta$ phase transition temperature. Around room temperature, the effect of the piezoelectric stiffening is a possible reason of the differences. As described above, the present study considers the piezoelectric stiffening, but it was ignored in the previous study. Therefore, reported values tend to be larger than the present values.

Around the phase transition temperature, the difference becomes larger than that at room temperature; for example, the difference in $C_{33}$ exceeds $15 \%$. This cannot be explained only by piezoelectric stiffening. A possible reason is the difference in the number of resonance frequencies measured in the experiment. In the ATAR method and the RUS method, reliability of resultant elastic constants is markedly improved as the number of resonance frequency increases. In the previous study, only 11 resonance frequencies were used around the phase transition temperature for the six $C_{i j}$, but the present study uses more than 22 resonance frequencies. Among independent elastic constants, $C_{44}$ is sensitive to the resonance frequencies, and it is determined accurately even if the number of resonance frequency is small. Therefore, the difference

TABLE I. Elastic constants $C_{i j}(\mathrm{GPa})$ at room temperature and elevated temperature, and first-order temperature coefficient $a_{i j}\left(\times 10^{-4} \mathrm{~K}^{-1}\right)$ of elastic constants of langasite and $\alpha$-quartz.

\begin{tabular}{ccccccccc}
\hline \hline & $i j$ & 11 & 12 & 13 & 14 & 33 & 44 & 66 \\
\hline \multirow{4}{*}{ Langasite } & $C_{i j}$ at $293 \mathrm{~K}$ & 188.6 & 104.7 & 95.6 & 14.4 & 258.9 & 53.3 & 42.0 \\
& $C_{i j}$ at $1224 \mathrm{~K}$ & 172.5 & 96.4 & 87.8 & 11.0 & 228.1 & 47.7 & 38.0 \\
& $a_{i j}$ & -0.90 & -0.98 & -0.99 & -2.66 & -1.29 & -1.04 & -0.85 \\
& $C_{i j}$ at $294 \mathrm{~K}$ & 86.8 & 7.0 & 11.8 & -18.0 & 105.4 & 58.2 & 39.9 \\
$\alpha$-quartz & $C_{i j}$ at $843 \mathrm{~K}$ & 72.0 & -27.4 & -3.4 & -13.4 & 79.9 & 41.3 & 49.7 \\
& $a_{i j}$ & -0.49 & -25.08 & -6.94 & 0.67 & -2.19 & -1.67 & 1.66 \\
\hline \hline
\end{tabular}




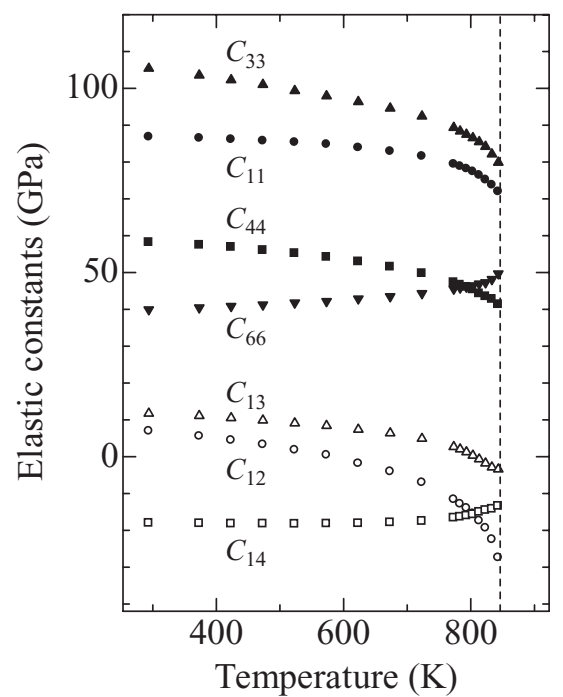

FIG. 6. Temperature dependence of elastic constants of $\alpha$-quartz. A dashed line shows the phase transition temperature of $846 \mathrm{~K}$.

in $C_{44}$ is small. However, other elastic constants are not determined as accurately as $C_{44}$, and the smaller number of the resonance frequencies significantly deteriorates the accuracy, causing larger differences. From the above discussions, we consider that the present study gives a more reliable set of elastic constants. Table I shows the first-order temperature coefficient of $C_{i j}$ of $\alpha$-quartz calculated between 294 and $373 \mathrm{~K}$.

A notable feature in the temperature dependence of elastic constants is the positive temperature dependence of a diagonal component $C_{66}$ of elastic constant matrix. Usually $C_{i j}$ decrease with increasing temperature. Although $C_{14}$ increases with increasing temperature, interpretation of off-diagonal component of elastic constant matrix is complicated, and it is not often essential. Here, we discuss its origin with the crystallographic structural evolution accompanying the thermal expansion. Before focusing on $C_{66}$, the general relation between the $C_{i j}$ and the structural change in $\alpha$-quartz is consid-

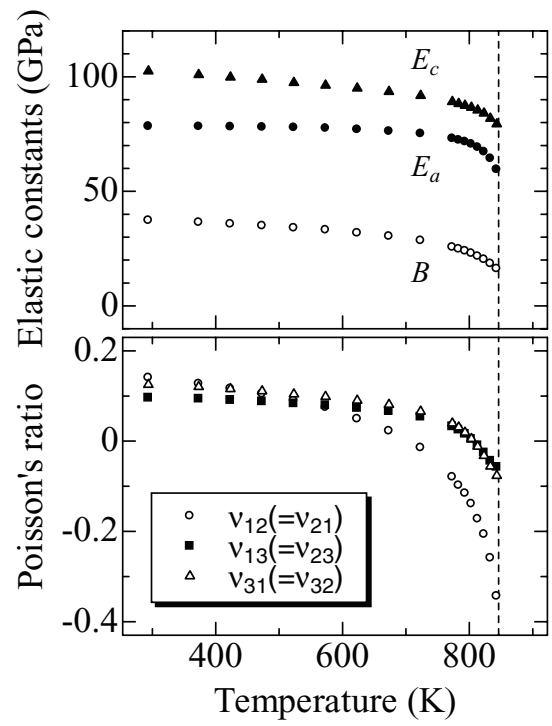

FIG. 7. Temperature dependence of Young's moduli, bulk modulus, and Poisson's ratios of $\alpha$-quartz.

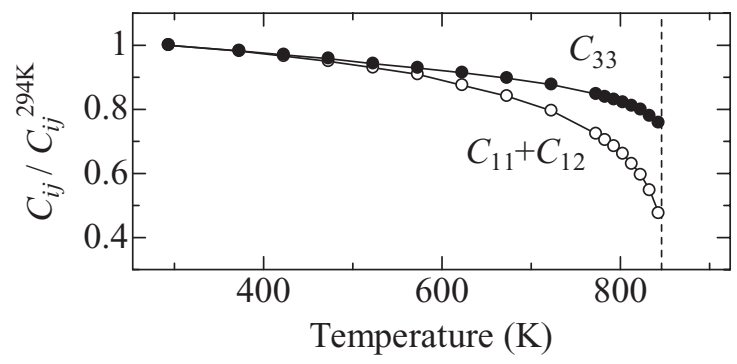

FIG. 8. Temperature dependence of the normalized in-plane stiffness $C_{11}$ $+C_{12}$ and out-of-plane stiffness $C_{33}$.

ered. In thermal expansion of $\alpha$-quartz, there is an anisotropy; the $a$ lattice parameter changes more than the $c$ lattice parameter. ${ }^{15}$ Considering that the thermal expansion changes elastic constants through the higher order elasticity, ${ }^{22}$ the anisotropy in the thermal expansion should cause anisotropy in the temperature dependence of the elastic constants as well. Figure 8 shows the in-plane elastic constant $C_{11}+C_{12}$ and the out-of-plane elastic constant $C_{33}$. $C_{11}+C_{12}$ denotes the inplane stiffness under the plane strain condition, $S_{3}=0$, where $S_{i}$ is the engineering strain. In the figure, $C_{11}+C_{12}$ changes more than $C_{33}$. This behavior is related with the difference in the thermal expansion coefficients; larger thermal expansion causes larger change in the elastic constants. Thus, this relationship supports the view that the temperature dependence of $C_{i j}$ is related with the crystallographic structural change.

The increment of $C_{66}$ by heating is now considered. To understand the detail of the structural change, local strain caused by temperature increment is calculated using the atomic positions measured by Kihara. ${ }^{23}$ Figure 9 compares the structure of $\alpha$-quartz at $298 \mathrm{~K}$ with that of $\beta$-quartz at $848 \mathrm{~K}$ (just above the phase transition temperature) together with the coordinate axes. As the temperature increases, the atoms in $\alpha$-quartz move toward the atomic positions of $\beta$-quartz. As seen in the figure, the deformation is never uniform. From the coordinate $u_{n}^{i}(T)$ of each atom at $T$ $=298 \mathrm{~K}$ and at elevated temperature $T$, strain component $\gamma_{i j}$ caused by heating is deduced by searching for $\gamma_{i j}$ that minimizes the mean-square difference between the coordinate at the elevated temperature and expected coordinates that each atom would have if they were uniformly deformed by heating, $D^{2}(T)=\sum_{n} \sum_{i}\left(u_{n}^{i}(T)-\sum_{j}\left(\delta_{i j}+\gamma_{i j}\right) u_{n}^{j}(298)\right)^{2}$ where the indices $i$ and $j$ denote spatial coordinates and the index $n$ runs over the atoms inside the spherical volume indicated by dashed circle with radius $r$ in Fig. 9. ${ }^{24}$ From the $\gamma_{i j}$ that minimizes $D^{2}$, the engineering strain $S_{i}$ is determined.

Figure 10(a) shows the calculated strain with different $r$ at $848 \mathrm{~K}$. As $r$ increases, $S_{1}$ and $S_{2}$ increase gradually and approach a specific strain. Similar behavior appears in $S_{3}$. Regarding $S_{4}$, fluctuation with $r$ is intense and it converges on zero as the temperature increases. On the other hand, $S_{5}$ and $S_{6}$ are zero. The fluctuations reveal that deformation by thermal expansion is never uniform. If structural change by heating were uniform, the strains would be constant without depending on $r$. When $r$ becomes significantly large, the macroscopic strain approaches the specific strain that is deduced from the change in the lattice constant in the $a$ and $c$ directions; macro- 


\begin{tabular}{|c|c|c|}
\hline & $\alpha$-quartz & $\beta$-quartz \\
\hline $\mathrm{Si}$ & $\bullet$ & $\bigcirc$ \\
\hline $\mathrm{O}$ & $\bullet$ & $\bigcirc$ \\
\hline
\end{tabular}

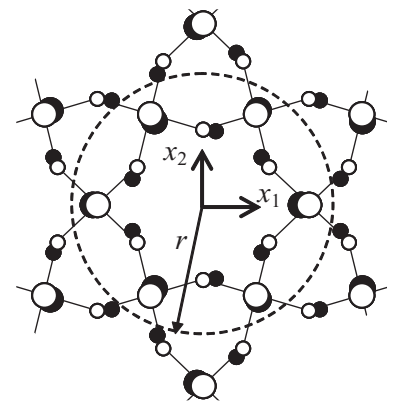

(a)

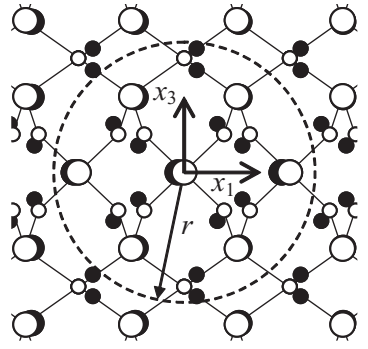

(b)
FIG. 9. Comparison of structure between $\alpha$-quartz at $298 \mathrm{~K}$ and $\beta$-quartz at $848 \mathrm{~K}$. (a) is a projection onto the $x_{1}-x_{2}$ plane and (b) is onto the $x_{1}-x_{3}$ plane.

scopically local shear strains are canceled and only longitudinal strain components remain. Also, strain evolution with temperature is calculated with $r=8 \AA$ in Fig. 10(b). In the figure, strain changes depending on the temperature, but $S_{5}$ and $S_{6}$ are unchanged. In these results, it is noted that local strain caused by heating is complicated but $S_{5}$ and $S_{6}$ are zero. Elastic softening near the phase transition is considered to decrease the strain energy that is required to achieve the transition. However, zero strain of $S_{6}$ indicates that softening of the corresponding stiffness $C_{66}$ is not necessarily required for decreasing the strain energy, and the observed nonsoftening of $C_{66}$ is acceptable. Regarding $S_{5}$, the same interpretation is applicable to $C_{55}\left(=C_{44}\right)$. However, $S_{4}$ is not locally zero, and softening of the corresponding stiffness $C_{44}$ is required. Thus, strain evolution caused by the thermal expansion provides a possible interpretation of the notable temperature dependence of $C_{66}$.

Another feature is the unusual decrease of Poisson's ratios toward negative values with increasing temperature. Keskar and Chelikowsky ${ }^{25}$ calculated Poisson's ratio of $\alpha$ quartz with uniaxial stress in the $c$ direction, and predicted

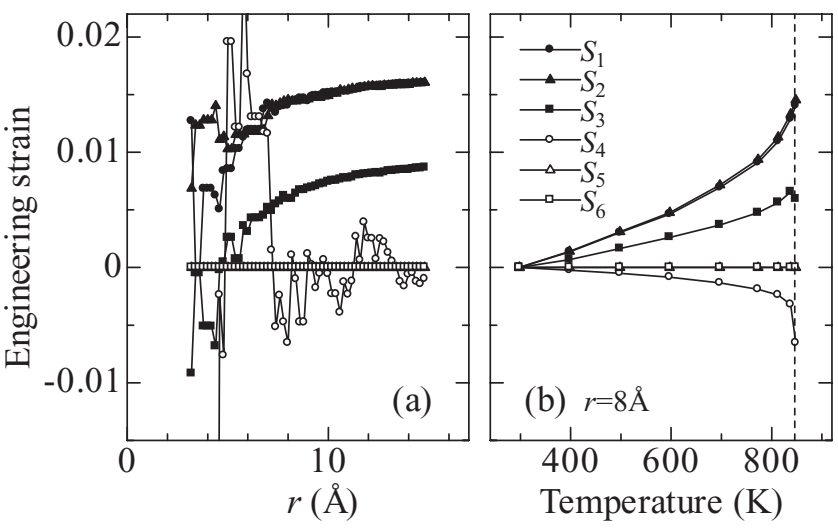

FIG. 10. Engineering strain caused by thermal expansion of quartz. (a) Relationship with the radius $r$ of the spherical region that strain is calculated at 848 K. (b) Strain evolution with temperature increment at $r=8 \AA$.

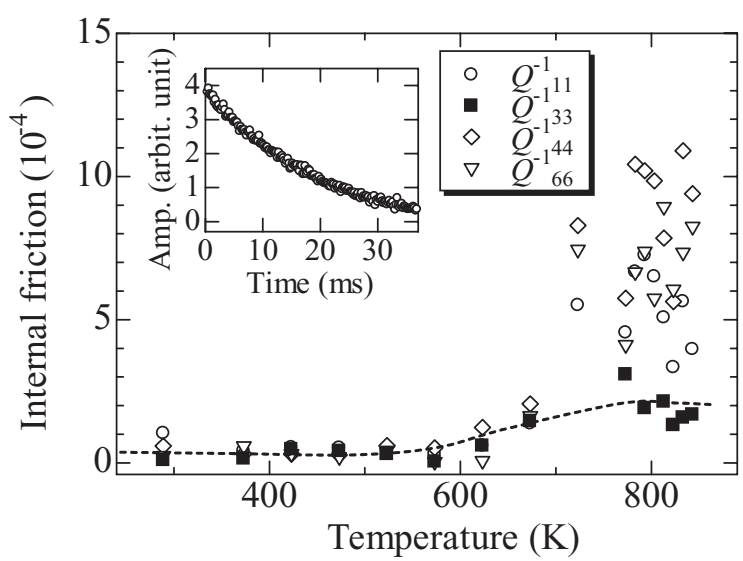

FIG. 11. Diagonal components of internal friction tensor of $\alpha$-quartz. A dashed line is drawn to guide the eye and represents temperature dependence of $Q_{33}^{-1}$. Inset shows decay curve of a resonance vibration of $0.771 \mathrm{MHz}$ at $573 \mathrm{~K}$.

negative value of Poisson's ratio when sufficiently large tensile stress is applied. Their prediction says that rigidity of $\mathrm{SiO}_{4}$ tetrahedra and its rotation contribute the negative Poisson's ratio, and lower mass density is required for this. Considering that thermal expansion decreases mass density, the observed negative values of Poisson's ratios should be explained in the same manner as that proposed for deformation under the uniaxial stress. The interpretation should be also applicable to negative values of $C_{12}$ and $C_{13}$ just below the transition temperature, because they denote relationship between deformations in orthogonal directions as Poisson's ratios denote, and their physical meaning seems to be similar to Poisson's ratio.

For evaluating internal friction, decay curve of vibrational amplitude at each resonance frequency was measured (inset in Fig. 11). Attenuation coefficient $\alpha$ was deduced by fitting an exponential function $A e^{-\alpha t}$ to the decay curve ( $A$ is a constant), and internal friction $Q^{-1}$ of each resonance frequency was calculated by $Q^{-1}=\alpha / \pi f$, where $f$ is a resonance frequency. Then, internal friction tensor $Q_{i j}^{-1}$ was calculated from $Q^{-1}$ values at individual resonances and the calculated contributions of elastic constants to the resonance frequencies. Detail of the calculation procedure is described elsewhere. ${ }^{14}$

Figure 11 shows the diagonal components of $Q_{i j}^{-1}$. As temperature approaches the phase transition temperature, internal friction becomes large for all components and an anisotropy appears between $Q_{11}^{-1}$ and $Q_{33}^{-1} ; Q_{11}^{-1}$ is larger than $Q_{33}^{-1}$. This indicates that acoustic vibrations related to $C_{11}$ attenuate faster than those related to $C_{33}$ at these temperatures. Similar anisotropy is seen in the lattice parameters. In $\alpha$-quartz, the thermal expansion coefficient in the $a$ direction is larger than that in the $c$ direction (Fig. 8(a)). Furthermore, in the $c$ direction, thermal expansion is interrupted around $798 \mathrm{~K}$. The interruption indicates that at this temperature thermal phonons related to $C_{33}$ are not stimulated by heating. If we assume the phonon-phonon interaction mechanism by Akhieser ${ }^{26}$ that thermal phonons disturbed by acoustic phonons remove energy from the acoustic phonons during returning to the equilibrium state as the major damping mech- 
anism, the loss of acoustic energy should be related closely to the thermal phonons. As seen in the thermal expansion coefficients, thermal phonons in the $a$ direction are preferentially excited by heating, and it enhances the interaction between the acoustic phonon and thermal phonons. On the other hand, in the $c$ direction, the interaction is not enhanced. Considering the interactions, anisotropy in the internal friction tensor is explained by the phonon-phonon interactions and the interruption in the thermal expansion coefficients.

\section{CONCLUSIONS}

Capability of the antenna transmission acoustic resonance method for high-temperature elastic constant was demonstrated by applying it to langasite and $\alpha$-quartz. In langasite, resonance frequencies were measured up to $1224 \mathrm{~K}$ using the antenna method, and temperature dependences of $C_{i j}$ were successfully determined. In $\alpha$-quartz, $C_{i j}$ were measured at high temperature just below the $\alpha-\beta$ phase transition temperature, and a difference was observed between the present values and the reported values. Origin of the unusual nonsoftening in $C_{66}$ was discussed considering the local strain caused by the thermal expansion, and it was found that $S_{6}$ is zero without depending on the local deformation and the softening of $C_{66}$ by heating does not necessarily contribute to reduction of elastic strain energy for the phase transition. In the internal friction, unexpected behavior was observed in the $c$ direction. It shows the relationship with the thermal expansion coefficient in the $c$ direction, and it was expected that the phonon-phonon interactions affect the thermal expansion as well as the internal friction.

Considering the advantages over other methods that a complete set of elastic constants is determined from a single specimen by an experiment and is applicable until the temper- ature at which the specimen's piezoelectricity disappears, the antenna transmission acoustic resonance method shows much promise.

${ }^{1}$ I. Koga, M. Aruga, and Y. Yoshinaka, Phys. Rev. 109, 1467 (1958).

${ }^{2}$ R. Bechmann, Phys. Rev. 110, 1060 (1958).

${ }^{3}$ M. Schulz and H. Fritze, Renewable Energy 33, 336 (2008).

${ }^{4}$ J. Kushibiki, I. Takanaga, and S. Nishiyama, IEEE Trans. Ultrason. Ferroelectr. Freq. Control 49, 125 (2002).

${ }^{5}$ I. Ohno, J. Phys. Earth 24, 355 (1976).

${ }^{6}$ A. Migliori, J. L. Sarrao, W. M. Visscher, T. M. Bell, M. Lei, Z. Fisk, and R. G. Leisure, Physica B 183, 1 (1993).

${ }^{7}$ I. Ohno, K. Harada, and C. Yoshitomi, Phys. Chem. Miner. 33, 1 (2006).

${ }^{8}$ J. R. Gladden, G. Li, R. Adebisi, S. Firdosy, T. Caillat, and V. Ravi, Phys. Rev. B 82, 045209 (2010).

${ }^{9}$ R. Tarumi, H. Nitta, H. Ogi, and M. Hirao, Philos. Mag. 91, 2140 (2011).

${ }^{10}$ H. Ogi, H. Ledbetter, S. Kim, and M. Hirao, J. Acoust. Soc. Am. 106, 660 (1999).

${ }^{11}$ J. Schreuer, J. Rupp, C. Thybaut, and J. Stade, Proc.-IEEE Ultrason. Symp. 1, 373 (2002).

${ }^{12}$ H. Ogi, K. Motohisa, T. Matsumoto, K. Hatanaka, and M. Hirao, Anal. Chem. 78, 6903 (2006).

${ }^{13}$ H. Ogi, H. Nagai, Y. Fukunishi, M. Hirao, and M. Nishiyama, Anal. Chem. 81, 8068 (2009).

${ }^{14}$ H. Ogi, N. Nakamura, K. Sato, M. Hirao, and S. Uda, IEEE Trans. Ultrason. Ferroelectr. Freq. Control 50, 553 (2003).

${ }^{15}$ R. J. Ackermann and C. A. Sorrell, J. Appl. Crystallogr. 7, 461 (1974).

${ }^{16}$ J. Kräußlich, S. Höfer, U. Zastrau, N. Jeutter, and C. Bachtz, Cryst. Res. Technol. 45, 490 (2010).

${ }^{17}$ G. F. Engel and P. W. Krempl, Phys. Lett. A 92, 469 (1982).

${ }^{18}$ V. G. Zubov, M. M. Firsova, and T. M. Molokova, Kristallografiya 8, 112 (1963).

${ }^{19}$ H. Fritze, J. Electroceram. 26, 122 (2011).

${ }^{20}$ H. Ogi, T. Ohmori, N. Nakamura, and M. Hirao, J. Appl. Phys. 100, 053511 (2006).

${ }^{21}$ W. P. Mason, "Properties and uses of quartz crystals," in Piezoelectric Crystals and Their Application to Ultrasonics (Van Nostrand, New York, 1950), pp. 78-113.

${ }^{22}$ Y. Hiki and A. V. Granato, Phys. Rev. 144, 411 (1966).

${ }^{23}$ K. Kihara, Eur. J. Mineral. 2, 63 (1990).

${ }^{24}$ M. L. Falk and J. S. Langer, Phys. Rev. E 57, 7192 (1998).

${ }^{25}$ N. R. Keskar and J. R. Chelikowsky, Nature (London) 358, 222 (1992).

${ }^{26}$ A. Akhieser, J. Phys. (USSR) 1, 277 (1939). 